\title{
Financial Derivative Uncertainty Claim Pricing Using Meixner Distribution Skewed Operators
}

\author{
Godswill U. Achi ${ }^{1}$, J. U. Okafor ${ }^{1}$, Ogwo Obiageri ${ }^{2}$ \& Solomon Okechukwu ${ }^{2}$ \\ ${ }^{1}$ Department of Mathematics, Abia State Polytechnic, Aba, Abia State, Nigeria \\ ${ }^{2}$ Department of Mathematics, Abia State University, Uturu, Abia State, Nigeria \\ Correspondence: Godswill U. Achi, Department of Mathematics, Abia State Polytechnic, P.M.B. 7166, Aba, Abia \\ State, Nigeria. Tel: 234-803-705-8344. E-mail: achigods@yahoo.com
}

Received: June 27, 2013 Accepted: July 22, 2013 Online Published: August 13, 2013

doi:10.5539/jmr.v5n3p69 URL: http://dx.doi.org/10.5539/jmr.v5n3p69

\begin{abstract}
In this paper, consideration is given to the problem of independent/uncertain claim pricing using distribution operators. This method was earlier studied in Insurance Pricing where the original operator was described in relation to normal distribution. We apply the Meixner process to financial pricing since the normal distribution is a very poor model to fit log-returns of financial assets like stocks or indices. For us to realize a better fit, we substitute the normal distribution by Meixner process. Hence, we generalize this approach by using an operator based on the density Meixner distribution. We further show how Meixner operator function can be used to derive the formular for asset pricing of independent/uncertain future returns of a risky asset.
\end{abstract}

Keywords: contingency claim pricing, distribution operators, Meixner process, normal distribution

2010 AMS Classification: 79M30, 97K69

\section{Introduction}

Of recent, Mathematics of Finance has gained huge respect on the bases of its effect on the financial industry. The foundation of financial mathematics as it is known today has its origin in the seminal papers by Black and Scholes (1973) and by Merton (1973), where the Ito's formula has been used for deriving a compact pricing formula for a standard European call option by formulating explicitly the model on the risk-neutral measure, under a set of assumptions.

Option valuation is one of the most important topics in financial mathematics. The exact modeling of financial price series is significant for the pricing and hedging of financial derivatives such as option. To price and hedge derivatives securities, it is vital to have a good modeling of the probability distribution of the underlying product.

The most well-known continuous time model used is the calibrated Black-Scholes model. It uses the normal distribution to fit the log-returns of the underlying; the price process of the underlying is given by the geometric Brownian motion

$$
S_{t}=S_{0} \exp \left(\left(\mu-\frac{\sigma^{2}}{2}\right) t+\sigma B_{t}\right) .
$$

Where $\left\{B_{t}, t \geq 0\right\}$ is a standard Brownian motion, i.e. $B_{t}$ follow a Normal distribution with mean 0 and variance $t$. It is known however that the log-returns of most financial assets have an actual kurtosis that is higher than that of the Normal distribution.

Empirical evidence has shown that the normal distribution is a very poor model to fit log-returns of financial asset such as stocks. In order to achieve a better fit we replace the Brownian motion by a special Levy process called the Meixner process. Several authors have proposed similar process models. Notable contributions were made, for instance, Luscher (2005) used the Normal Inverse Gaussian (NIG) to price synthetic Collateralized Debt Obligations (CDO), Barndorff-Nielsen (1995) proposed the NIG Levy process, Eberling and Keller (1995) proposed the Hyperbolic models and their generalizations, Wang (2000) proposed pricing based on a normal-based distribution operator for a form of insurance risk, Osu and Achi (2012) price contingent claim using distortion operators by Cauchy distribution under a simple transformation. 
In this paper, we show how Meixner operator function can be used to derive the formula for asset pricing of uncertain future returns of a risky asset. More so, we generalize this approach by using an operator based on the density Meixner distribution.

\subsection{Meixner Process}

The Meixner process is a Levy process $\{X(t)\}_{t \geq 0}$ that has Meixner distributed increments. Specifically, $X(t)$ has a Meixner $(x: a, b, d t, m t)$-distributed with parameters $a>0,-\pi<b<\pi, d>0$ and $m \in \mathbb{R}$. The density of the Meixner distribution Meixner $(a, b, d, m)$ is given by

$$
f_{\text {meixner }}(x ; ; a, b, d, m)=\frac{(2 \cos (b / 2))^{2 d}}{2 a \pi \Gamma(2 d)} \exp \left\{\frac{b(x-m)}{a}\right\}\left|\Gamma\left(d+\frac{i(x-m)}{a}\right)\right|^{2}
$$

where $\Gamma$ denotes the Gamma function.

The characteristic function of Meixner $(a, b, d, m)$ is given by

$$
\emptyset_{\text {meixner }}(x ; a, b, d, m)=E[\exp (i u X)]=\left(\frac{\cos (b / 2)}{\cosh \frac{a u-i b}{2}}\right)^{2 d} \exp (i m u) .
$$

A Meixner $(x: a, b, d t, m t)$-distributed random variable has the following stylized features (and compared to the Normal distribution):

$\begin{array}{lccc} & \text { Meixner }(a, b, d, m) & \operatorname{Normal}\left(\mu, \sigma^{2}\right) & \text { Meixner }(a, 0, d, m) \\ \text { Mean } & m+\operatorname{adtan}(b / 2) & \mu & m \\ \text { Variance } & \frac{a^{2} d}{2}\left(\cos ^{-2}(b / 2)\right) & \sigma^{2} & \frac{a^{2} d}{2} \\ \text { Skewness } & \sin (b / 2) \sqrt{2 / d} & 0 & 0 \\ \text { Kurtosis } & 3+\frac{3-2 \cos ^{2}(b / 2)}{d} & 3 & 3+\frac{1}{d}\end{array}$

See (Schoutens, 2002) on more information about Meixner process.

\section{Time Changed Levy Process}

Let $(\Omega, f, p)$ be a probability space in this work; a random variable representing net income or profit after some time and $h$ be a constant. We presume that for any random variable described on the probability space, its characteristic function exists. That is, for any random variable

$$
E\left[e^{i h x}\right]<\infty
$$

For the cumulative distribution, (cdf), $F_{X}(\cdot)$ with differential $d F_{X}(\cdot)$, corresponding to a given random variable $X$, we define by

$$
d F_{X}^{(i h)}(x)=\frac{e^{i h x} d F_{X}(x)}{E\left[e^{i h X}\right]} .
$$

where the characteristic function of $\mathrm{X}$ under

$$
p^{E S S M M}=p_{X[0 . T] h^{*}}^{E S S}
$$

$\emptyset_{t}^{E S S M M}(u)$, is defined by

$$
\emptyset_{t}^{E S S M M}(u)=E_{p(E S S M M)}\left[e^{i u X_{t}}\right]=\frac{E_{p}\left[e^{i u X_{t}} e^{h^{*} X_{t}}\right]}{E_{p}\left[e^{h^{*} X_{T}}\right]} .
$$

The Levy-Khintchine formula for the characteristic function of $X_{t}$ is;

$$
\emptyset_{X(t)}(u) \equiv E\left[e^{i u X_{t}}\right]=e^{-t \varphi_{x}(u)} t \geq 0, u \in R^{d} .
$$

Where the characteristic exponent, $\varphi_{X}(u), u \in R^{d}$ is given by

$$
\varphi_{x}(u)=-i \mu^{T} u+\frac{1}{2} u^{T} \Sigma u-\int_{R^{d}-\{0\}}\left(e^{i u^{T} x}-1-i u^{T} x 1_{|x|<1}\right) \pi(d x) .
$$


The characteristic function of the time changed Levy-process $Y_{t} \equiv X_{t}$ is

$$
\emptyset_{y}(u) \equiv E e^{i u^{T} X_{t}}=E\left[E\left[e^{i u^{T} X_{T}} / f_{t}^{v}\right]\right]=E\left[E\left[e^{i u^{T} X^{T}} / T_{t}=\theta\right]=E e^{-T_{t}} \square_{x}(u)=\mathcal{L}_{T}\left(\square_{x}(u) .\right.\right.
$$

That is the Laplace transform of the stochastic time $T_{t}=\int_{0}^{t}(v(s-) d s$ evaluated at the characteristic exponent of X.

\subsection{The General Case of Correlated Time Changed}

More generally, the general Fourier transform, CF, of $Y_{t} \equiv X_{T_{t}}$ under measure can be represented as the "Laplace Transform" of $T_{t}$ under a new complex-valued measure $\emptyset(u)$, calculated at the characteristic-exponent $\varphi_{x}(u)$ of $X_{t}$.

$$
\emptyset_{Y_{t}}(u) \equiv E\left[e^{i u^{T} Y_{t}}\right]=E^{u}\left[e^{-T_{t} \square_{x}(u)}\right] \equiv \mathcal{L}_{T_{t}}^{u}\left(\square_{x}(u)\right) .
$$

For each $u \in D, \emptyset(u)$ is absolutely with respect to $p$ and is defined by

$$
E \frac{d \emptyset(u)}{d p} /_{f_{t}} \equiv M_{t}(u) \equiv \operatorname{esp}\left(i u^{T} Y_{t}+T_{t} \square_{x}(u) \quad u \in D\right.
$$

which is a complex valued $p$-Martingale with respect to $\left\{f_{T_{t t \geq 0}}\right\}$ for each $u \in D$.

Why is $M_{t}(u) \equiv E \frac{d \emptyset(u)}{d p} / f_{T_{t}}=\exp \left(i u^{T} Y_{t}+T_{t} \square_{x}(u), u(t)\right)$ a $p$-Martingale?

Remark 1 The familiar Wald Martingale defined on a Levy process

$$
Z_{t}(u) \equiv \exp \left(i u^{T} X_{t}+t \square_{x}(u)\right)
$$

Time changed (ie replacing t by $T_{t}$ ) preserves the Martingality.

Proof.

$$
E\left[e^{i u^{T} Y_{t}}\right]=E\left[e^{i u^{T} Y_{t}+T_{t x}(u)-T_{t x}}\right]=E\left[M_{t}(u) e^{-T_{t x}(u)}\right]=E^{u}\left[e^{-T_{t x}(u)}\right] \equiv \mathcal{L}_{T_{t x}}^{u}((u) .
$$

While the validity of mathematical operation is constant, the multi-rated measure loses whatever could be its merit.

\section{Existence Theorem of Esscher Martingale Measure (ESSMM) for Geometric Levy Process}

We believe in the existence of what that follows, since the Martingale condition for an Esscher changed expectation measure $\emptyset=P_{Z[0, T]}^{E S S}, h$ is

$$
E_{\emptyset}\left[e^{-r} X_{t}\right]=e^{-r} X_{0} E_{\emptyset}\left[e^{z_{t}}\right]=e^{-r} X_{0} \frac{E_{p}\left[e^{(h+1) z_{t}}\right.}{E_{p}\left[e^{h z_{t}}\right]}=X_{0}
$$

This state equals the situation as follows

$$
E_{p}\left[e^{(h+1) Z_{t}}\right]=e^{r} E_{p}\left[e^{h Z_{t}}\right]
$$

and this equals also the following statement

$$
\begin{gathered}
\emptyset\left(-i(h+1)=e^{r} \emptyset(-i h),\right. \\
\emptyset(u)=E_{p}\left[e^{i u Z_{t}}\right] .
\end{gathered}
$$

where $\emptyset(u)$ is the characteristic function of $Z_{t}$.

To develop the existence theorem, setting

$$
f^{\operatorname{ESSMM}}(h)=b+\left(\frac{1}{2}+h\right) \delta^{2}+\int_{|x| \leq 1}\left[\left(e^{x}-1\right) e^{h x}-x\right] V(d x)+\int_{|x|>1}\left(e^{x}-1\right) e^{h x} V d x
$$

Then we have

Theorem 1 Existence Condition For ESSMM.

If the equation

$$
f^{E S S M M}=r,
$$


has a solution $h^{*}$, then the ESSMM of $X_{t}, P^{E S S M M}$, exists and

$$
P^{E S S M M}=P_{z[0, T]^{\prime} h^{*}}^{E S S M M}=P_{Z_{T}, h^{*}}^{E S S}
$$

The process $Z_{T}$ same as a Levy process under $P^{E S S M M}$ and the generating triplet of $Z_{t}$ under $P^{E S S M M},\left(\delta^{(E S S M M)^{2}}\right.$, $\left.V^{E S S M M}(d x), b^{E S S M M}\right)$ is

$$
\begin{gathered}
\delta^{(E S S M M)^{2}}=\delta^{2}, \\
V^{E S S M M}(d x)=e^{h^{*} x} V(d x) \\
b^{E S S M M}=h+h^{*} \delta^{2}+\int_{|x \leq 1|} x\left(e^{h^{*} x}-1\right) V d x .
\end{gathered}
$$

Proof. The Equation (3.5) is equivalent to (3.3). Therefore, $P_{Z[0, T], h^{*}}^{E S S M M}$ is a Martingale measure of $X_{t}$. The characteristic function of $Z_{t}$ under $P_{z[0, T], h^{*}}^{E S S M M}=\emptyset_{t}^{E S S M M}$ is by definition

$$
\emptyset_{t}^{E S S M M}(u)=E_{p(E S S M M)}\left[e^{i u z_{t}}\right]=\frac{E_{p}\left[e^{i u z_{t}} e^{h^{*} z T}\right]}{\left.E_{p\left[e^{h^{*} z} T\right.}\right]} .
$$

and this is equal to

$$
\frac{E_{p}\left[e^{\left(i u+h^{*}\right) z_{t}}\right]}{E_{p}\left[e^{h^{*} z_{t}}\right]}=\frac{\emptyset_{t}\left(u-i h^{*}\right)}{\emptyset_{t}\left(-i h^{*}\right)}
$$

By simple calculation, we obtain;

$$
\begin{aligned}
\emptyset_{t}^{E S S M M}(u)= & \exp \left\{t \left(-\frac{1}{2} \delta^{2}+i\left(b+h^{*} \delta^{2}+\int_{|x| \leq 1} x\left(e^{h^{*} x}-1\right) V(d x)\right.\right.\right. \\
& \left.+\int_{|x| \leq 1}\left(e^{i u x}-1-i u x\right) e^{h^{*}} V(d x)+\int_{|x>1|}\left(e^{i u x}-1\right) e^{h^{*} x} V(x)\right\}
\end{aligned}
$$

This formula proves the result of the theorem.

\subsection{Price of a European Call Option}

Theorem 2 For a probability space $(\Omega, f, P)$, a random variable $X_{T}$ which is the net income or profit at after some time. And for any $X_{T}$ defined on the probability space, its characteristic function exits, i.e, for any random variable $X: \Omega \rightarrow C^{d}, E\left[e^{i h X}\right]<\infty, i h \in C^{d}$.

Where $C$ is the complex plane and the characteristic function of $X_{T}$ has the form

$$
\emptyset_{X_{t}}(h) \equiv E\left[e^{\left(i h^{T} X_{t}\right)}\right]=e^{\left(t \Psi_{X}(h)\right)}, \quad t \geq 0
$$

with the option price

$$
C=e^{(-r)} X_{o} N\left(\left(i \mu-\ln \left(\frac{K}{X_{o}}\right)\right) / \delta\right)+((1+h) x) e^{i \mu+\frac{1}{2} \delta^{2} h+\delta^{2} \theta}-\left(e^{(-r)} K\left(i \mu-\ln \left(K / X_{o}\right)\right) / \delta .\right.
$$

Proof. Assuming that the stock prices are log-normally distributed and that the stochastic process

$$
C=e^{-r t} \int_{-\infty}^{\infty} \operatorname{maz}\left(S_{t}-K, 0\right) f(x, t ; i h) d x
$$

Let the stochastic process $\left\{X_{t}\right\}$ be a Weiner process with mean per unit time $\mu$ and variance per unit time $\delta^{2}$. Then

$$
F(x, t)=N\left(x ; i \mu t, \delta^{2} t\right)
$$

and

$$
\begin{gathered}
m(z, t)=e^{\left(i \mu z+\frac{1}{2} \delta^{2} x^{2}\right) t}, \\
m(z, t ; h)=e^{\left[\left(i \mu+h \delta^{2}\right) Z+\frac{1}{2} \delta^{2} x^{2}\right] t}, \\
F(x, t ; h)=N\left(x ;\left(i \mu+h \delta^{2}\right) t, \delta^{2} t\right),
\end{gathered}
$$




$$
\begin{gathered}
C=e^{-r t} \int_{-\infty}^{\infty}\left(S_{0} e^{x}-K\right) \frac{e^{i h x}}{m(i h, t)} f(x, t) d x, \\
m(i h, t)=E\left[e^{i h x}\right]=e^{h t\left(-i \mu x+\frac{1}{2} \delta^{2} x^{2}\right)}, \\
C=\frac{e^{-r t}}{e^{\left(-i \mu \theta+\frac{1}{2} \theta^{2}\right)}}\left[X_{0} \int_{-\infty}^{\infty} e^{(1+h) x} f(x, t) d x-K \int_{-\infty}^{\infty} e^{h x} f(x, t) d t\right] .
\end{gathered}
$$

Using Equation (3.13a) to find the value of $x$ which determines the region of integration,

$$
\delta e^{(1+h) x} e^{-h t\left(-i \mu \theta+\frac{1}{2} \theta^{2}\right)} \geq 0 .
$$

We have

$$
(i+h) x-\frac{1}{2} \delta^{2} \theta^{2} h+i \mu \theta=\ln \left(\frac{K}{S_{0}}\right)
$$

which is equivalent to the well-known Kolmogorov Backward Equation in (Goovaerts \& Laeven, 2008) with the drift function modified for the change of probability measure as proved by the Esscher transform.

Thus,

$$
C=e^{-r} X_{0} N\left(\frac{i \mu-\ln \left(\frac{K}{X_{0}}\right)}{\delta}\right)+(1+h) x e^{i \mu+\frac{1}{2} \delta^{2} h+\delta^{2} \theta}-e^{-r} K\left(\frac{i \mu-\ln \left(\frac{K}{X_{0}}\right)}{\delta}+(1+h) x\right) .
$$

From Equations (3.11) and (3.13b), we can find $h^{*}$ for a random variable normally distributed $X_{t}^{2}$,

$$
h^{*}=\frac{r-i \mu}{\delta^{2}}-\frac{1}{2}
$$

Replacing in (3.15) we obtain,

$$
C=X_{0} N\left(\frac{\left(-\ln \left(\frac{K}{X_{0}}\right)+\left(r+\frac{1}{2} \delta^{2}\right)\right)}{\delta}\right)-e^{-r} K N\left(\frac{\left(-\ln \left(\frac{K}{X_{0}}\right)+\left(r-\frac{1}{2} \delta^{2}\right)\right)}{\delta}\right)
$$

Thus from the characteristic function of the time changed Levy process, we obtained the traditional Black-Scholes formula for pricing a European call option. Note that the expected rate of return $i \mu$, which represents the preferences of investors, does not appear in the last formula.

\section{Meixner and Option Pricing}

Let $\emptyset^{m}$ denotes the Meixner distribution function

$$
\emptyset^{m}=\frac{(2 \cos (b / 2))^{2 d}}{2 a \pi \Gamma(2 d)} \exp \left\{\frac{b(x-m)}{a}\right\}\left|\Gamma\left(d+\frac{i(x-m)}{a}\right)\right|^{2}
$$

We define the Meixner operator as $H\left(x, \lambda^{m}\right)=\int_{0}^{\infty} h_{x} S_{x}(x) d x=\emptyset^{m}\left[(k)+\lambda^{m}\right]$.

Proposition 3 Consider the Meixner operator $\int h_{x ; a, b, m, d}\left(S_{x}(X)\right) d x$. Let $z$ be a variable and let $X=h(z)$ be a transformation through a continuous function $h$, then $\emptyset^{m}=f(x ; ; a, b, m, d)=\operatorname{mex}(x ; a, b, m, d)=>f(x)$ and $S(x)$ is the decumulative function of $f(x)$.

Proof. Given

$$
\begin{gathered}
S(x)=1-f(x)=P(x>t) \\
S(x)=1-\operatorname{mex}(x ; a, b, m, k)=1-\emptyset^{m}(x ; a, b, m, k)
\end{gathered}
$$

By definition, we have

$$
H\left(x, \lambda^{m}\right)=\int_{0}^{\infty} h_{x} S_{x}(x) d x=\emptyset^{m}\left[(k)+\lambda^{m}\right] \text { for every } m \in R
$$

Where

$$
\begin{aligned}
S_{X}(t) & =P[x>t]=P[h(z)>t] \\
& =P\left[\frac{h(z)}{h}>t / h\right]=P\left[z>h^{-1}(t)\right] \\
& =1-\emptyset^{m}\left[h^{-1}(t)\right] \\
& =\emptyset^{m}\left[-h^{-1}(t)\right]=d
\end{aligned}
$$


If we apply now the operator function $h_{(x, a, b, m, k)}$ on $S_{x}$, we have

$$
\begin{gathered}
h_{(x, a, b, m, k)} S_{x}(t)=\emptyset^{m}\left[(k)+\lambda^{m}\right] \\
\emptyset^{m}\left[\left(\left[-h^{-1}(t)\right]\right)+\lambda^{m}\right]=\emptyset^{m}\left[-h^{-1}(t)+\lambda^{m}\right]=1-\emptyset^{m}\left(h^{-1}(t)-\lambda^{m}\right)
\end{gathered}
$$

Applying the probability again we have that $P\left[z>h^{-1}(t)-\lambda^{m}\right]$, normalizing

$$
\begin{gathered}
P\left[\frac{z-b}{a}>\frac{h^{-1}(t)-\lambda^{m}-b}{a}\right]=p\left[\frac{z-b}{a}>\frac{h^{-1}(t)-\lambda^{m}-b}{a}\right] \\
P\left(x>\frac{h^{-1}(t)-b-\lambda^{m}}{a}\right) \\
P\left(x a>h^{-1}(t)-b-\lambda^{m} a\right)
\end{gathered}
$$

Note: If $x=\frac{z-b}{a}=x a=z-b \Rightarrow z=x a+b$

$$
\begin{gathered}
P\left(x a+b+\lambda^{m} a>h^{-1}(t)\right) \\
P\left[z+\lambda^{m} a>h^{-1}(t)\right]
\end{gathered}
$$

Multiplying through by $h$, we have

$$
\begin{gathered}
P\left[h\left(z+\lambda^{m} a\right)>h^{-1}(t) \cdot h\right]=P\left[h ( z + \lambda ^ { m } a > t ] \Rightarrow E ^ { n } \left[h\left(z+\lambda^{m} a\right)\right.\right. \\
E_{x}^{n}=E^{n}\left(h\left(z+\lambda^{m} a\right)=\int h_{x} S_{x}(x) d x=E\left[S_{0} e^{2+\lambda^{m} a}\right]\right.
\end{gathered}
$$

Where $E^{n}$ denotes the expectation under the density measure n, then

$$
H\left(S_{T},-\lambda\right)=E\left[S_{0} e^{2+\lambda^{m} a} T\right] \text { for every } m \in R
$$

This result shows that under a $h_{x, a, b, m, d}$ operator, a meixner random variable is translated by a factor $\lambda^{m} a$. This generalizes the equivalent result found in NIG.

The reason for every $m \in R$, is that, stochastically, the market is changing over time by making business time more stochastic. The $m$ incorporates the stochastic volatility effect and the resulting option prices can be determined very similarly to empirical prices. $S_{T}$ evolves like a risk neutral asset as $\lambda^{m}$ is calliberated to verify the risk neutral condition even as $m$ tends to nor $\infty$.

- Then the expression for $H\left[S_{T},-\lambda\right]$ simplifies to $H\left(S_{T}-\lambda^{m}\right)=S_{0} e^{r T}$.

Here, under meixner operator with value $\lambda^{m}$ gives $\emptyset^{m}\left[(u)+\lambda^{m}\right]$, the price at $S_{T}$ evolves like a risk neutral asset because $\lambda^{m}$ is calibrated to verify the risk neutral condition.

Hence

$$
\begin{gathered}
H\left(S_{T},-\lambda\right)=S_{0} e^{r T}=\int h_{x, a, b, m, k}\left(S_{x}(t)\right) d t=\emptyset^{m}\left[(u)+\lambda^{m}\right]=E\left[S_{0} e^{2+\lambda^{m} a}\right]=E\left[h\left(z+\lambda^{m} a\right)\right] \\
\Rightarrow H\left[S_{T},-\lambda\right]=E\left[S_{0} e^{2 t-\lambda^{m} a} T\right] .
\end{gathered}
$$

- More generally, the proposition can be extended to the case when the security price is a function of a symmetrically distributed random variable.

- If we apply the price $E\left[z+\lambda^{m} a\right]$, the capital required for the standard European call payoff is

$$
H\left[F\left(S_{T}, k\right) ; \lambda\right]=E\left[h\left(2 T+\lambda^{m} a\right]=\left[S_{0} e^{2 t+\lambda^{m} a}-k\right]=\int_{-\infty}^{\infty}\left(S_{0} e^{2}-k\right) \operatorname{mex}\left(x: a, b, m, k+\lambda^{m} a\right) d t .\right.
$$

Having known the density function $\mathrm{H}\left(F\left(S_{T}\right)\right)$ of the stock price at the expiring time, $S_{T}$ under the risk neutral measure $\lambda^{m} a$ we can easily prize European call and put option by simply calculating the expected value (Schoutens, 2003). 


\subsection{Contingent Claim Pricing}

Let us look at the Meixner asset price model $\emptyset^{m}\left[(U)+\lambda^{m}\right]$ and a standard European call option pay-off at maturity time $T$ given by $f\left(S_{T}, K\right)=\left(S_{T},-K\right)$. This is purely a function of the $\left(f_{T}, p\right)$-random variable $S_{T}$ that becomes the change for the security offered at the time $T$. It can be stated as $S_{T}=h\left(z_{T}\right)$ for a function $h(z)=\left(S_{0} e^{z}-K\right)$, the value at time zero is therefore given by the expectation of the payoff under the Martingale measure.

$$
\begin{gathered}
C(k, T)=E_{Q}\left[\exp ^{(-r T)} \max \left(S_{T}-k\right), 0\right] \\
\exp ^{(-r T)} \int_{0}^{\infty} g_{Q}(S, T) \max (S,-K) d s \\
e^{-r T} \int_{k}^{0} g_{Q}(S, T)(S-k) d s \\
\exp ^{(-r T)} \int_{0}^{\infty} g_{Q}\left(S_{T}\right) s d s-k\left(\exp ^{-r T}\right) \pi
\end{gathered}
$$

Where $\pi$ is the probability under $\emptyset$ of the future prize of the risky asset $\left.\int_{Q b}^{\infty}\left(S_{0} e^{z}-k\right) \frac{k}{a} z^{k-1} e^{-z k}+\lambda^{m} a\right) d z$. Then $H\left[F\left(S_{T}, k\right)-\lambda^{m}\right]$ becomes

$$
\begin{gathered}
\left.\int_{Q b}^{\infty}\left(S_{0} e^{z}-k\right) k z^{k-1} e^{-z k}-\lambda^{m}\right) d z \\
\int_{Q b}^{\infty} S_{0} e^{z} \cdot k z^{k-1} e^{-z k}-\lambda^{m} d z-k \int_{Q \alpha}^{\infty} k z^{k-1} e^{-z}-\lambda^{m} d z
\end{gathered}
$$

If we set $\lambda^{m}$ to be the one that make $S_{T}$ evolve like a risk neutral asset i.e. $\lambda^{m}=\frac{\pi-r_{c}}{a} T$.

This shows that the price assessed with the pricing function related to the Meixner operator with parameter $\lambda^{m}$ is given by

$$
\begin{aligned}
& S_{0} e^{z} \int_{Q b}^{\infty}\left[x, k, a_{T}\left(b+\lambda^{m}\right) T d z-k \int \operatorname{mex}\left(x, k, a_{T}\left(b+\lambda^{m}\right) T d z\right.\right. \\
&= S_{0} e^{z}\left(1-\emptyset_{b}^{m}-\lambda^{m}\right)-\left(1-\emptyset_{b}^{m}-\lambda^{m}\right) \\
&= S_{0} e^{z}\left(\emptyset_{b}^{m}+\lambda^{m}\right)-k\left(\emptyset_{b}^{m}+\lambda^{m}\right) \\
& e^{-r T} H\left(F\left(S_{T}, k\right),-\lambda^{m}\right)=S_{0} \operatorname{mex}\left[\emptyset_{b}, x, k, a\left(b+\lambda^{m}\right)\right] T-k e^{-r T} \operatorname{mex}\left[\emptyset_{b}: x, k, a_{T}\left(b+\lambda^{m}\right) T\right.
\end{aligned}
$$

which is the prize at time zero i.e. the price at $S_{t}$. Therefore

$$
H\left(X ; \lambda^{m}\right)=\int_{0}^{\infty} P\left[h\left(Z+\lambda^{m}\right)>t\right] d t=E\left[h\left(z+\lambda^{m}\right)\right]=S_{0} e^{z_{T}}+a .
$$

If $S_{t}$ is the price of a security at time $t$, following a geometric Brownian motion

$$
S_{t}=S_{0} e^{\left(\mu-\frac{\sigma^{2}}{2}\right) t+\sigma W_{t}}
$$

where $W_{t}$ is a Brownian motion under $p$ then $S_{T}$ can be written as a function of the standard normal random variable $\mathrm{Z}$. Here,

$$
S_{T}=h(Z)
$$

where

$$
h(x)=S_{0} e^{\left(\mu-\frac{\sigma^{2}}{2}\right) T+\sigma \sqrt{T} x}
$$

Applying the function we have

$$
\begin{gathered}
H\left(S_{T}, \lambda^{m}\right)=E\left[h\left(z-\lambda^{m}\right)\right] \\
E\left(S_{0} e^{\left(\mu-\frac{\sigma^{2}}{2}\right) T+\sigma \sqrt{T \lambda^{m} a}-\sigma \sqrt{T} x}\right)=S_{0} e^{\left(\mu-\frac{\sigma^{2}}{2}\right) T-\sigma \sqrt{T \lambda^{m} a}+\frac{\sigma^{2}}{2}}
\end{gathered}
$$

for $b=\frac{\pi-r_{c}}{\sigma} \sqrt{T}=\lambda^{m} a T=\lambda$.

Simplifies to

$$
H\left(S_{T},-\lambda\right)=S_{0} e^{-\lambda^{m} a} T
$$


then the current price becomes

$$
e^{-\lambda^{m} b} T H\left(S_{T}, \lambda^{m}\right)=e^{-\lambda^{m} a} T S_{0} e^{-\lambda^{m} a} T=S_{0} .
$$

Therefor, the parameter $\lambda^{m}$ measure the obvious discount equals the for seen security charge to the substantive security charge considering the continual market price variations in order to make business time more stochastic. The option price result incorporates these stochastic volatility effects which is determined very similarly to empirical prices. If we consider the pay-off of an European call option (with maturity $T$ and strike price $k$ ) we have

$$
S_{T}=C\left(S_{T}, K\right)=\left(S_{T},-K\right) .
$$

Where $S_{T}$ is a lognormal random variable. Applying the function to this payoff with

$$
b=\frac{\pi-r_{c}}{\sigma} \sqrt{T}=\lambda^{m} a T .
$$

We can show that

$$
\begin{gathered}
e^{-\lambda^{m} T} H\left[C\left(X_{T}, k\right):-\lambda\right]=S_{0} \phi\left(\ln \left(\frac{x_{0}}{k}\right)+\left(\frac{\lambda^{m}+\theta^{2}}{2}\right) T-\theta \sqrt{T}\right) \\
e^{-\lambda^{m} T} k \phi \frac{\ln \left(\frac{x_{0}}{k}\right)+\left(\frac{\lambda^{m}+\theta^{2}}{2}\right) T}{\sigma \sqrt{T}}=S_{0} \phi\left[\ln \left(\frac{x_{0}}{k}\right)+\left(\frac{\lambda^{m}+\theta^{2}}{2}\right)-e^{-r T} k \phi \frac{\ln \left(\frac{x_{0}}{k}\right)+\left(\frac{\lambda^{m}+\theta^{2}}{2}\right) T-\sigma \sqrt{T}}{\sigma \sqrt{T}}\right] .
\end{gathered}
$$

\section{Conclusion}

$\lambda^{m}$ is the risk free rate for every $m \in R$, which shows that as market changes over time, it becomes more stochastic and the parameter $m$ incorporates these stochastic volatility effect making the option price determine very similarly to empirical prices as it fit log-returns of financial assets like stock or indices.

In other words, under meixner operator with value $\lambda^{m}=\frac{\mu-r_{c}}{a} T \ln \emptyset^{w}\left[(k)+\lambda^{m}\right]$ the prize at $S_{T}$ evolves like a risk neutral asset because $\lambda^{m}$ is calibrated to verify the risk neutral condition.

Consequently, we assumed that the prize of return at $S_{T}$ follows a meixner distribution in place of normal distribution this is because for us to achieve a better fit we replace the normal distribution by meixner distribution. Hence, we generalize this approach by using an operator based on the density meixner distribution. The meixner family allows for heavier and skewed tail, as the kurtosis of normal distribution is always less than that of meixner distribution kurtosis.

\section{References}

Achi, G. U. (2012). Financial Derivative Pricing under Probability Distortion Operator via Esscher Transformation (Ph.D thesis, Abia State University, Nigeria).

Barndorff-Nielson, O. E. (1995). Normal Inverse Gaussian distribution and modeling of stock returns. Research Report No 300, Department of Theoretical Statistics, Aarhus University.

Black, F., \& Schole, M. (1973). The Pricing of Options and corporate liabilities. Journal of Political Economy, 81, 637-654. http://dx.doi.org/10.1086/260062

Carr, P., \& Wu, L. (2004). Time Changed Levy process and Option pricing. Journal of Financial Economics, 71, 113-141. http://dx.doi.org/10.1016/S0304-405X(03)00171-5

Eberlein, E., \& Keller, U. (1995). Hyperbolic distribution in finance. Bernoulli, 1(3), $281-299$. http://dx.doi.org/10.2307/3318481

Goovaerts, M. J., \& Laeven, R. J. A. (2008). Actuarial Risk measure for financial derivatives pricing. Insurance: Mathematics and Economics, 42(2), 540-547. http://dx.doi.org/10.1016/j.insmatheco.2007.04.001

Grigelionis, B. (1999). Process of Meixner type. Lith. Maths. J., 39(1), 33-41. http://dx.doi.org/10.1007/BF02465533

Harmada, M., \& Sherries, M. (2003). Contingent Claim Pricing using probability distortion operators: method from Insurance Risk pricing and their relationship to Financial theory. Applied Mathematical Finance, 10, 19-47. http://dx.doi.org/10.1080/1350486032000069580 
Luscher, A. (2005). Synthetic CDO pricing using double Normal Inverse Gaussian copula with stochastic factor loadings. (Diploma thesis submitted to the ETA ZURICH and UNIVERSITY of ZURICH for the degree of Master of Advanced Studies in Finance). Retrieved from http://www.javaquant.net/paper/AnnelisLuescher.pdf

Merton, R. C. (1973). Theory of Rational option pricing. Bill Journal of Economic and Management Science, 4, 141-183. http://dx.doi.org/10.2307/3003143

Olschowka, K. (2007). The Meixner Process. Seminar on alternative models for stock prices.

Osu, B. O., \& Achi, G. U. (2013). Contingent Claim Pricing using the Cauchy Probability Distortion Operator under simple Transformation. International Journal of Aplied Physics and Mathematics, 3(1). http://dx.doi.org/10.7763/IJAMP.2013.V3.164

Osu, B. O., \& Achi, G. U. (2012). Risk Neutral Option Pricing via Esscher Transformation using Characteristics Function. International J. of Maths. Sci. \& Engg. Appls. (IJMSEA), 6(v), 373-383.

Schoutens, W. (2003). Levy Process in finance: pricing financial derivatives. John Wiley and Sons Ltd.

Schoutens, W., \& Leuven, K. U. (2002). The Meixner Process: Theory and Applications in Finance. Celestijnenlaan 200B B-3001 Leuven, Belgium. Retrieved from http://perswww.kuleuven.be/ u0009713/004wsreport.pdf

Schoutens, W. (2002). The Meixner Process: Theory and Applications in Finance. EURANDOM Report 2002004, EURANDOM, Eindhoven.

Wang, S. (2000). A class of Distortion Operator for pricing finance and Insurance risks. Journal of Risk and Insurance, 36, 15-36. http://dx.doi.org/10.2307/253675

\section{Copyrights}

Copyright for this article is retained by the author(s), with first publication rights granted to the journal.

This is an open-access article distributed under the terms and conditions of the Creative Commons Attribution license (http://creativecommons.org/licenses/by/3.0/). 